\title{
Searching for Evidence-Based Medicine in the Literature Part 2: Resources
}

\section{Keywords:}

Evidence-based medicine; Computer-assisted instruction; Internet
Reprint Requests:

Barbara A. Bartkowiak, MLIS, MST

George E. Magnin Medical Library

Marshfield Clinic

1000 N. Oak Avenue

Marshfield, WI 54449

Tel: 7 I5-389-4285

Fax: $715-389-5366$

Email: bartkowiak.barbara@mcrf.mfldclin.edu

Received: December 10, 2004

Accepted: January 4, 2005
Barbara A. Bartkowiak, MLIS, MST

$\mathrm{n}$ an effort to seek out evidence-based medicine (EBM), a bit of preparation is required prior to the literature search. The first requirement is formation of a well-built clinical question and necessitates focusing the query into the PICO (an acronym for patient/population, intervention/exposure, comparisons, and outcomes) format. After careful sculpting of this clinical question, the task becomes a selection of the most appropriate information resources and the search execution itself.

One of the easiest to use websites for consideration in the EBM selection process is freely available at the US National Library of Medicine's PubMed/MEDLINE database (http://www.ncbi.nlm.nih.gov/entrez/query.fcgi). A series of PubMed services are listed in the border on the left side of the main screen. Within the Clinical Queries feature are two methods (filters) for extracting EBM materials from the PubMed database: Clinical Queries using Research Methodology Filters and Systematic Reviews. The Clinical Queries using Research Methodology Filters method is geared toward clinicians and uses an extensive strategy in the background based on research by R. Brian Haynes ${ }^{1}$. Clinicians may select either sensitivity (results will include the most relevant articles but will probably include some less relevant ones) or specificity (results will include the most relevant articles but will probably omit a few) within one of the categories: therapy, diagnosis, etiology, or prognosis. A search term can then be entered into the query box at the bottom of the page and the search begun. The search results will include combinations of indexing terms and text words required by EBM methods. The second method available on the PubMed site is Systematic Reviews in which one can locate systematic reviews, meta-analyses, reviews on clinical trials, and similar publication types. The Limits feature of the PubMed database can be used to refine the retrieval. Limits is a time-saving feature that quickly restricts your literature search with options you select from provided listings of age groups, genders, human or animal populations, languages, or type of article. The Limits feature is located immediately below the search term entry box at the top of the search page. In either the Clinical Queries using Research Methodology Filters or Systematic Reviews sections, ease of use is primary. It is a perfect resource for a "quick and dirty" EBM search: the price is right; the execution is simple; the results based solidly in EBM methodology. Although limited to journal literature 
retrieval, the PubMed Clinical Queries service is a manageable and reliable beginning in the EBM search process.

The next best resources are the web sites of EBM organizations. The Cochrane Library (http://www.thecochranelibrary.com) is proprietary, but is considered one of the foremost in the EBM field. Other proprietary websites that specialize in EBM include Bandolier (http://www.jr2.ox.ac.uk/bandolier/index.html), Clinical Evidence

(http://www.clinicalevidence.com/ceweb/conditions/index.jsp), and TRIP Database Plus (http://www.tripdatabase.com/). The difficulty, however, is access. While these products provide value-added features such as literature selection beyond journal articles, a standard methodology for literature inclusion, and formats that reflect EBM emphases, there are subscription fees required for access, and the search engines may be difficult to use.

There are free web sites that assist in the deliberation of what other resources are available. Netting the Evidence - A ScHARR Introduction to Evidence Based Practice on the Internet (http://www.nettingtheevidence.org.uk/) offers a robust listing of EBM resources with annotations and links. The listings are extensive but do not always indicate which resources are free. The site has not been updated since March 2004 and some of its resources may have moved. Andrew Booth, a central UK educational figure in EBM, is listed as the site's contact person.

Generally, searching for EBM resources can be disparate, but happily, the US National Library of Medicine and Netting the Evidence at the University of Sheffield's School of Health and Related Research (http://www.shef.ac.uk/scharr) offer methods to assist in the search process. The quality of information that can be extracted with a rigorous EBM methodology in the literature using one of these two sites can give a more accurate and substantive result than a hit-or-miss approach to searching.

After selection of EBM resources, the search execution depends on the resource used. While the US National Library of Medicine's Clinical Queries feature takes care of the search strategy for you, another website that will help determine what a solo MEDLINE strategy should include is available from the University of Illinois at Peoria's Library of the Health Sciences (http://uic.edu/depts/lib/lhsp/resources/ebm.shtml). The section on Sample Medline Searches provides a template for searching articles in PubMed for therapy, diagnosis, prognosis, and etiology. The PubMed/MEDLINE search system is provided by various commercial vendors, other than at the US National Library of Medicine web site, and may vary in their abilities to refine the search strategy. This template can be useful in adapting terms and functions to the nuances of those engines. As such, it illuminates some of the correct terminology and combinations that constitute an EBM approach regardless of the provider.
Selection of an EBM resource and the search itself can be daunting, but it is possible to investigate the literature with some ease through reputable websites. Depending on one's financial resources, the array of EBM resources is flexible. The determinant factor, however, is the time and energy that is required to retrieve relevant, pragmatic information that is suitable for individualized patient care. The assessment of information requires another step in the EBM process, one that analyzes the retrieval with a more rigorous approach, and will be the focus of the final part of this series to appear in the May 2005 issue of Clinical Medicine \& Research.

\section{References}

1. Haynes RB, Wilczynski N, McKibbon KA, Walker CH, Sinclair JC. Developing optimal search strategies for detecting clinically sound studies in MEDLINE. J Am Med Inform Assoc. 1994;1:447-458.

\section{Author Affiliations}

Barbara A. Bartkowiak, MLIS, MST, George E. Magnin Medical Library, Marshfield Clinic, 1000 N. Oak Avenue, Marshfield, Wisconsin 54449. 